\title{
Provider time allotment tracking tool to effectively manage assignment commitments
}

\author{
Yu-Li Huang*1, Narges Shahraki ${ }^{1}$, Erin M. Wallin ${ }^{2}$, Eric W. Klavetter ${ }^{2}$, Kyle W. Klarich ${ }^{3}$ \\ ${ }^{1}$ Robert D. and Patricia E. Kern Center for the Science of Health Care Delivery, Mayo Clinic, Rochester, Minnesota, United \\ States \\ ${ }^{2}$ Department of Cardiology, Mayo Clinic, Rochester, Minnesota, United States \\ ${ }^{3}$ Department of Cardiovascular Diseases, Mayo Clinic, Rochester, Minnesota, United States
}

Received: March 4, 2021

DOI: $10.5430 /$ jha.v10n3p10
Accepted: April 29, 2021

Online Published: May 10, 2021

\begin{abstract}
Due to the rising demand with limited health service capacity, managing available resources effectively becomes an important task to reduce patient care delays and avoid unnecessary and costly capacity expansions. At the same time, staff satisfaction and/or burnout is a complementary consideration when designing optimal schedules. Deviation from the scheduled plan can cause delays in patient access and may lead to unsatisfaction among providers. Balancing demand management, staff satisfaction and generating optimized schedules quickly reveals the need for a tool that tracks provider time allotment over time, especially for the academic healthcare organization where providers are committed to multiple assignments, clinical and non-clinical. This tracking tool should allow management to proactively adjust allotment to unplanned changes in the schedule and increase participation. In this study, a tool is developed to track monthly provider assignments for the Department of Cardiovascular Medicine at Mayo Clinic, Rochester. The proposed tool produces two key outputs for each provider and assignment: 1) the recommended target workdays and 2) workday upper and lower bounds to accommodate for variability. This tracking tool is successfully implemented with implementation criteria, and the feedback is positive. The tool pulls the data systematically from the Mayo data platform and performs the necessary analysis on the data. It also automatically updates the values for the recommended target as well as upper and lower bounds for the remaining months in a year based on changes in the schedule so that provider commitment can be met at the end of year.
\end{abstract}

Key Words: Tracking tool, Health care, Time allotment, Assignment, Cardiovascular

\section{INTRODUCTION}

In recent years, demand for health care services increased as a result of the aging population and the emphasis on preventive cares. ${ }^{[1,2]}$ Drivers of physician burnout are largely rooted within healthcare organizations and systems and include lack of input or control for physicians with respect to issues affecting their work lives. ${ }^{[3]}$ Due to the rising demand and the capacity constraint, managing the available resources effectively becomes an important task to reduce patient care delays and avoid unnecessary and costly capacity expansions. ${ }^{[4,5]}$ Efficient use of available resources is a common challenge that almost all hospitals are facing. It is critical to not only reduce access delays but also increase the medical staff satisfaction to sustain the quality of care. ${ }^{[6]}$ Managing medical staff workload and simultaneously considering their satisfaction is not an easy task and is considered

\footnotetext{
*Correspondence: Yu-Li Huang; Email: huang.yuli@mayo.edu; Address: Robert D. and Patricia E. Kern Center for the Science of Health Care Delivery, Mayo Clinic, Rochester, Minnesota, United States.
} 
as an extremely challenging problem. ${ }^{[7]}$

The workload assignment problem is mainly studied in literature in terms of staffing and scheduling. The staffing problem is defined as the number of personnel needed in each shift to meet the demand, while the scheduling problem defines the individual physicians that need to be assigned to each shift to meet the staffing level. ${ }^{[8]}$ Accurately estimating the demand for physician workload is a key factor in developing a successful staffing/scheduling model. There are different drivers for uncertain demand which include emergencies, ${ }^{[9]}$ surgical duration $^{[10]}$ and patients who do not attend their scheduled appointments or no-shows. ${ }^{[11]}$ Some studies incorporate demand uncertainty in their models, but most of these studies are focused on the emergency department. ${ }^{[10,12]}$ However, there is uncertain demand in the other health care departments as well. ${ }^{[10]}$ In addition, the majority of the studies in the literature view demand as deterministic, which neglects the realistic physician demand profile. ${ }^{[13]}$

There is extensive literature on staffing and scheduling. ${ }^{[14-19]}$ However, there is less attention in the literature regarding how managers can keep track of providers' time allotment or how a tool could assist in managing physician schedules, and equitably assigning clinical and non-clinical time as well as vacation and other time away. This is essential to help manage burnout and balance workload. A tracking providers' time allotment tool is needed particularly when the demand is treated as deterministic in the staff/scheduling model. The tool is necessary to keep track of the assignments and make the necessary adjustments when there are any unpredicted changes on demand.

This paper describes the design of an algorithm to track providers' schedule in the Mayo Clinic Department of Cardiovascular Medicine (MCDCM). This tracking tool is to ensure provider task assignments are according to the scheduled plan and if not, be proactive and adjust in time for the future schedule, so that assignment commitment can be met at the end of the year. The manual tracking process prior to the tool implementation was time consuming and resulted in suboptimal decisions. The proposed framework can potentially be applied to other healthcare specialty settings as well as beneficial to other industries where a decision maker or a manager wishes to keep track of scheduled assignments over time.

\subsection{Current state}

MCDCM has 10 divisions, some of which are interventional, circulatory failure, ischemic, heart rhythm, and structural. There are approximately 200 providers, and each has their own assignment time commitment. Due to the large number

Published by Sciedu Press of providers, the department management team is in need to have a system to track the scheduled assignments; especially when it comes to equitable distribution of time between practice assignments such as clinic, hospital services and labs and the awarded non-clinical time such as administrative time, education, and research time. Administration work consists of being a division chair, physician lead, and more. Education is for teaching at medical school, running the accredited fellowship and residency programs since Mayo Clinic is a teaching hospital. Lab is where procedures take place such as transcatheter aortic valve replacement, echocardiograms, nuclear studies, catheter ablations and pacemaker replacement. Hospital service is where providers are assigned either physically round in hospital or are on call. Research work includes clinical trials, basic laboratory research, translational research, innovation, grant proposal and manuscript writing for scholarly publication. Providers at the beginning of each year have their time distribution to each assignment defined. This distribution also includes away times such as weekends, paid time off (PTO), and research trips. The current system captures these away times precisely for compensation purposes but allows providers to submit their times for other assignments freely without providing where they stand at that time. This results in missing the commitment for assignments at the end of year. Figure 1 indicates how much the interventional provider or interventionalist group deviated from the time commitment at the end of 2019. There are 11 providers in this group denoted from A to K. Each provider at minimum allots time to clinic, lab, hospital and research assignments. Only eight out of 11 providers have administration assignments and five out of 11 providers have education assignments. Most providers overly allot their time on clinic work, as much as 38 days, while research and hospital service are behind. The variation of time allotments among department providers is quite high, some are way behind while others are over their allocations. In fact, the department did not actually have a system to track providers' time allotted to each assignment. Therefore, the goal is to develop a tool that tracks providers' commitment monthly in terms of number of workdays for each task assignment and provides recommended target workdays as well as upper and lower bounds to accommodate for variability.

\section{Methods}

The tool is developed based on providers away time. Once the away time is realized, the time allotment for each month is calculated and distributed evenly throughout each month in relation to available workdays. The time committed for each assignment is incorporated in the model to ensure relative proportion for each assignment is built in each month so that 
the workload for each assignment is balanced. The upper and lower bounds of the time allotment for each assignment is also developed to provide flexibility in consideration of provider preference. This tool dynamically recalculates the
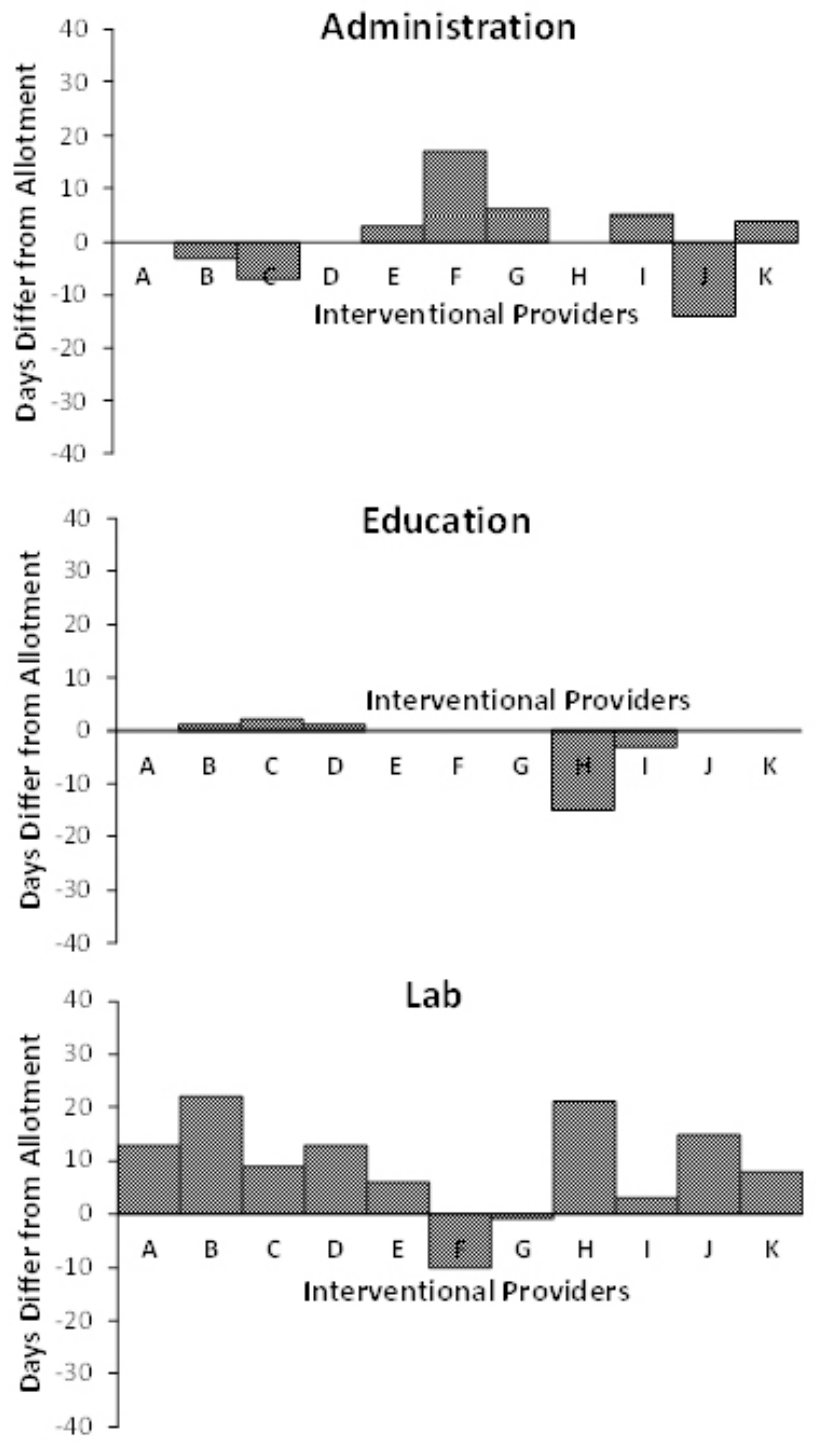

target and bounds for future months to reflect the times being allotted currently. The implementation criteria are then developed to ensure that the tracking tool is used appropriately for assignment time allotment.
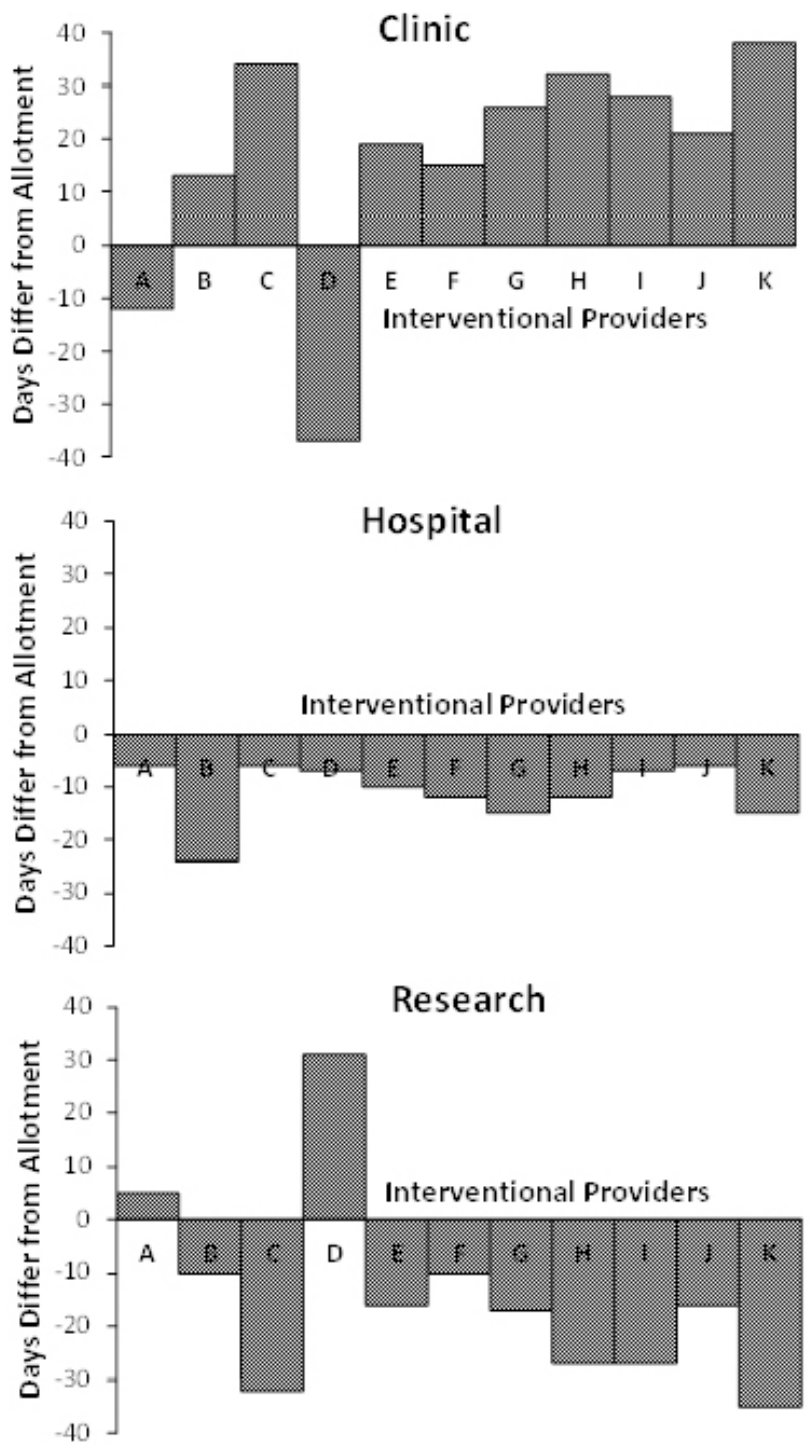

Figure 1. Days difference from assignment allotment for Interventional provider A-K in 2019

\subsection{Tool modeling}

The tracking tool is modeled as the following:

$A_{j}=$ Days in a month $j$, where $j=1,2,3, \cdots, 12$. For example, $A_{1}=31$ (January), $A_{2}=28$ (February), $A_{3}=31$ (March)... etc.

$B_{j}=$ Away days in month $\mathrm{j}$ (based on Mayo Clinic Clairvia database) including weekends, trips, and vacations. Clairvia is a Cerner-based system for workforce management and is currently being used by the MCDCM to build providers schedules.

$C_{j}=$ Possible workdays in month $j=A_{j}-B_{j}$
$D_{j}=$ Possible workdays for the rest of year after the previous month $j-1=\sum_{1}^{12} C_{j}$ $E_{j}=$ Percentage of workdays dedicated in month $j=\frac{C_{j}}{D_{j}}$ $F_{i}=$ Percent commitment for the assignment $i$ where $i=a, r$, $e, c, l, h . a=$ administration, $r=$ research, $e=$ education, $c=$ clinic, $l=$ lab, $h=$ hospital.

$G_{i}=$ Total committed assignment $i$ workdays in a year $=$ $210 \times m \times F_{i}$. The 210 days are standard workdays for a full-time provider $(m=1.0)$ and are calculated by total days in a year minus weekends, trips, vacations, and any paid time off. For providers who have joint appointments with other 
departments or are not full-time, the value $m$ varies. If $m=$ 0.8 , then the standard workdays are $210 \times 0.8=168$ days.

$H_{i, j}=$ Actual workdays for assignment $i$ in month $j$

$K_{i, j}=$ Remaining assignment $i$ commitment in month $j=$ [ $\left.K_{i, j-1}-H_{i, j-1}\right]$, where $K_{i, 1}=G_{i}$

$T_{i, j}=$ Target workdays for assignment $i$ in month $j=K_{i, j} \times$ $E_{j}$

An example for February $(j=2)$ target for an Interventionalist in administration $(i=\mathrm{a}): A_{2}=$ Days in February $=28$

$B_{2}=$ Away days in February $=8.5$

$C_{2}=$ Possible workdays in February $=28-8.5=19.5$

$D_{2}=$ Possible workdays in 2019 after January $=\sum_{2}^{12} C_{j}=$ 186

$E_{2}=$ Percentage of workdays dedicate in February = $19.5 / 186=10.5 \%$

$F_{a}=$ Percent commitment for the administration $=10 \%$

$G_{a}=$ Committed administration workdays in a year $=210 \times$

$10 \%=21$

$H_{a, 1}=$ Actual workdays for administration in January $=2$

$K_{a, 2}=$ Remaining administration in February $=K_{a, 1}-H_{a, 1}$ $=21-2=19, K_{a, 1}=G_{a}=21$

$T_{a, 2}=$ Target for administration workdays in February $=19$ $\times 10.5 \% \approx 1.9$

Lower bound $\left(L_{i, j}\right)$ for assignment $i$ in month $j$ is $1-p \%$ below the target, $T_{i, j}$. Hence, $L_{i, j}=T_{i, j} \times p \%$. The percentage $p$ should be chosen by practice and the smaller of $p$ the tighter the bound is. The minimum lower bound is zero.

Upper bound $\left(U_{i, j}\right)$ for assignment $i$ in month $j$ is the minimum of the followings:

$T_{i, j} \times(1+p \%), p \%$ above the target.

$K_{i, j}-\left(K_{i, j}-H_{i, j}\right) \times p \%$, remaining assignment $i$ in month $j$ minus the sum of the expected lower bound for the remaining year, meaning that the remaining months all perform at lower bounds. This is to prevent providers from overcommitting for a particular month in the remaining months of a year.

Continuing the example, assuming $p \%=50 \%$ and the actual workdays for administration in February $H_{a, 2}=0.5$ day, the lower bound $\left(L_{a, 2}\right)=T_{a, 2} \times p \%=1.9 \times 50 \%=1$ day and the upper bound $\left(U_{a, 2}\right)=\min \left[T_{a, 2} \times(1+p \%), K_{a, 2}\right.$ $\left.-\left(K_{a, 2}-H_{a, 2}\right) \times p \%\right]=\min [1.9 \times(1+50 \%), 19-(19$ $-0.5) \times 50 \%]=\min [2.9,9.8]=2.9$ days. The example indicates the target is about two days, and the provider can choose administration days between one and three days.

\subsection{Implementation criteria}

The proposed tool provides the allotment target and flexibility between upper and lower bounds. After time away becomes known, the total possible time in a month is fixed. If a time allotment deviates from its target for an assignment, it may prevent the allotment for other assignments from meeting the bounds. For instance, if a provider decides to have more days assigned to clinic work than the suggested target, this provider may have to designate less time on research to accommodate the difference. Hence, the actual allotment decision for each assignment should be constrained by the following criteria:

Criterion 1: $L_{i, j} \leq H_{i, j} \leq U_{i, j}$ for all $i$, meaning actual allotment for each assignment should be within upper and lower bounds

Criterion 2: $\sum_{i} H_{i, j} \leq C_{j}$ for all $i$, meaning sum of actual allotment for each assignment should be, at most, equal to possible workdays in month $i$

Given that these two criteria are met, the decision on time allotment for each assignment can still be quite flexible. For example, a full-time interventionalist dedicates $20 \%$ to research, $40 \%$ to lab, $14 \%$ to clinic, and $26 \%$ to hospital services. This provider in the first month is away for 14 days including weekends and holidays for a total of 17 (31 - 14) workdays. The recommendations for his/her lower bound, target, upper bound workdays are $(1.7,3.4,5.6)$ for research, $(3.4,6.8,10.2)$ for lab, $(1.2,2.4,3.6)$ for clinic, and $(2.2,4.4$, 6.6) for hospital. The target values sum up to be $17(3.4+6.8$ $+2.4+4.4$ ) days. One possible recommendation is to assign five days for research, seven days for lab, two days for clinic, and three days for hospital to fulfill 17 workdays (criterion 2) and each time allotment is within the upper and lower bounds (criterion 1). There are many other combinations to choose from given the bounds, and it is always best to be around the target.

\section{Results}

The tool modeling details were validated by providers and other key staff from the MCDCM. It was implemented for planning future assignment time allotment for each provider in the coming year.

\subsection{Tool outputs}

There are two primary outputs for this tool. One being reports that consist of what had happened and the recommendation and the second is visual presentations for a provider. Figure 2a demonstrates administration assignment, for example, for five providers in September 2020. To be noted, provider C in May, did five days when the lower bound is 5.5 days as well as provider $\mathrm{F}$ in June where actual day allotment is two days but the lower bound is suggested to be 2.8 days. The deviation outside of bounds is allowed to happen as long as the recalculation of the target and bounds, for the future months ensures the commitment is fulfilled at the end. Figure $2 b$ demonstrates visuals of what had been allotted in administra- 
tion assignment and what is suggested moving forward for a provider. The left panel indicates four months have passed and this provider followed quite closely to the target value each month; the dip in August is due to vacation. The visual on the right panel tracks where your accumulated time allotment of 19.5 days to April in relation to the administration commitment of 63 days in a year.

(a)

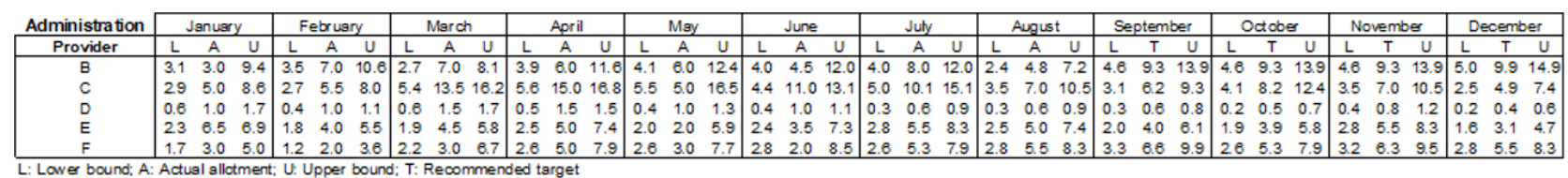

(b)
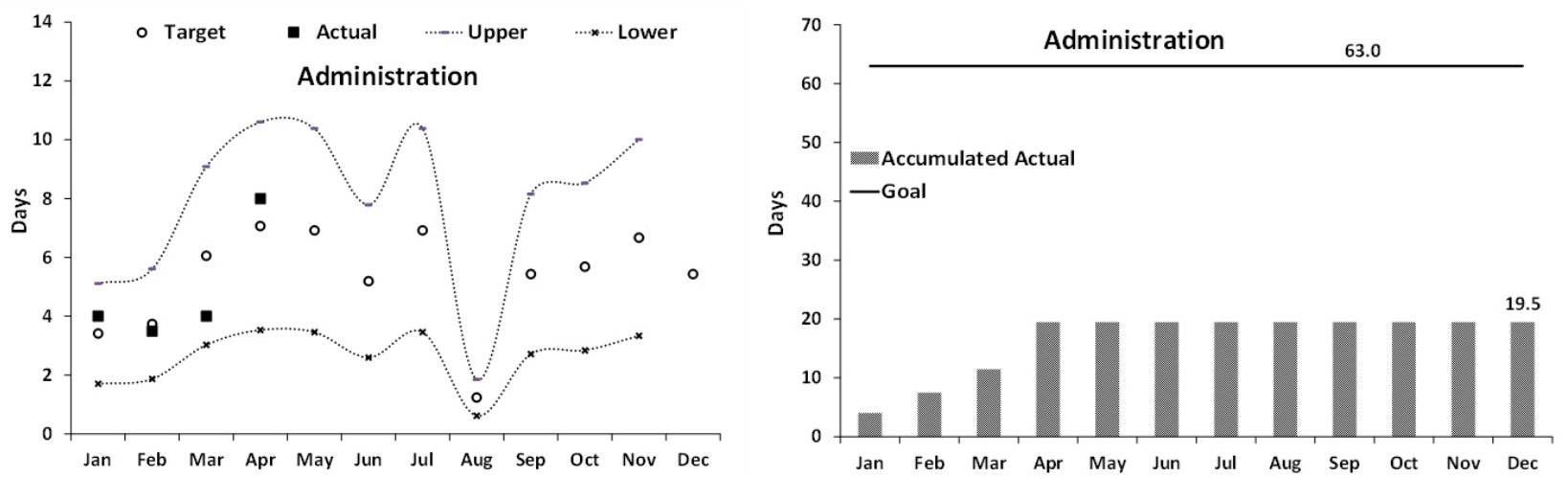

Figure 2. Administration report for five providers (a) and visual tracking for a provider (b)

\subsection{Implementation feedback}

Prior to the assignment tracker, the Medical Staff Assignment Coordinator (MSAC) team manually maintained an Excel spreadsheet to keep track of non-clinical time. The spreadsheet included all the consultants within the MCDCM, the total amount of non-clinical time they were allocated for the year, and the amount of days they were allocated each month. At the beginning of every month, the MSAC team would run reports in the Clairvia system to show the number of days of administration, research, and education time each consultant had in the previous month. The team would then manually enter the amount of days on the spreadsheet for each consultant. A formula was built into the spreadsheet to show if the team was negative or positive for assigned days for the previous month. By the time the team was able to manually enter the information onto the spreadsheet, the consultant schedules were already built, which limited the team to make changes to the schedule and give the consultant more or less non-clinical time.

With the assignment tracker, the MSAC team can be proactive in assigning non-clinical time by looking ahead and anticipating the amount of time that should be assigned per consultant and will no longer need to manually enter the information from Clairvia onto the spreadsheet. The assignment tracker will run after selecting a refresh button at the beginning of each month to pull the data in from Clairvia from the previous month. The team will receive the information more quickly and adjust the schedule before the schedule is built out for the year. Overall, the ability to run projected data gives the MSAC team the ability to adjust as they build the schedule.

\section{Discussion}

It is ideal to allot time, as the tracking tool suggested, monthly. However, when providers choose not to follow the tool, the impact not only significantly deviates from the assignment commitment at the year-end, but also disrupts patient access to care.

\subsection{Allotment impact}

When a provider exceeds an assignment in a month, there are two negative consequences. The first consequence is this provider may not be able to perform this assignment for the coming months. Figure 3 a shows a provider allotted his lab time more than the tool suggested in February, April, June, and July. This causes the tool to recommend minimal lab time for the rest of year. When there is a need for lab time during the rest of year, this provider must re-allocate other assignment times to the lab, which could result in other assignments not meeting commitment by the year end. Hence, the second consequence is the extra lab time this provider 
exceeded for the first seven months now needs to be taken allotment for the rest of year. This case in hospital service from other assignments. Figure $3 b$ indicates this provider had four out of seven months without performing hospital duty which causes the tool to suggest more hospital time also indicates the impact of under-allotment early drives high allotment later. These instances often occurred when there was not a tracking tool existing.

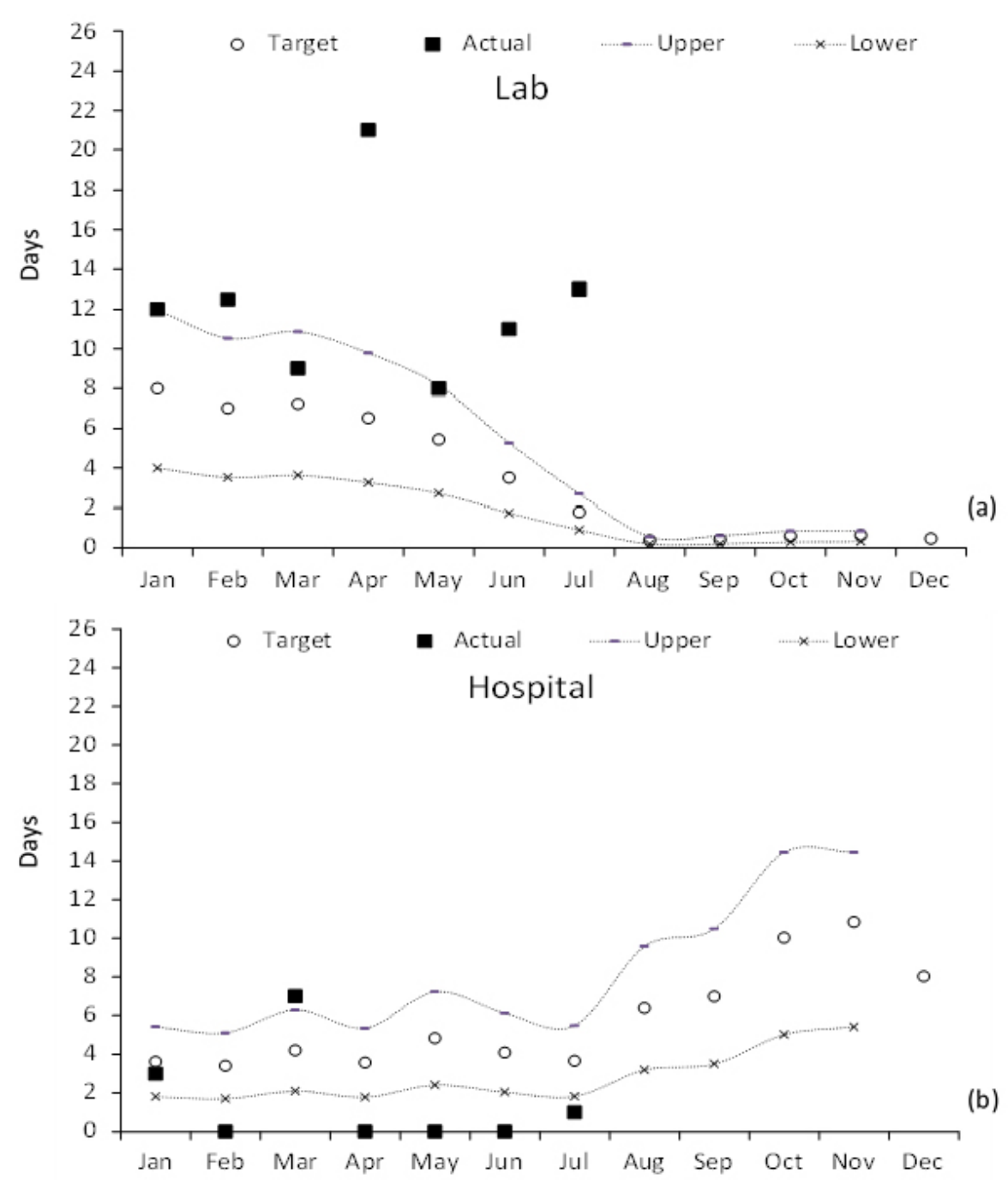

Figure 3. An example of over-allotment impact other assignments (a) lab and (b) hospital service

\subsection{Division level tracking}

The best practice is to track the individual provider for their time allotment on each assignment so that their commitment can be met at the end. However, providers are allowed to switch assignments such as clinic, lab, and hospital services, as long as practice needs are fulfilled. For example, if the hospital needs an interventionalist to be on call for the next week, it may be one of several interventionalists. To balance their clinical and nonclinical time assignments, tracking individual providers could reduce the flexibility for provider preferences. Tracking at the division level is also an option. When tracking at division level, the formulations in the Methods section are the same but need to roll up to the number of Published by Sciedu Press providers $w$, where $w=1,2, \cdots, n$, in a division. For example, let $A_{w j}^{d}=$ days in a month $j$ for provider $w$ in a division $d$. Therefore, days in a month $j$ for a division $d$ should be: $A_{j}^{d}$ $=\sum_{w}^{n} A_{w j}^{d}$. The same modification also applies to other parameters such as away days, possible workdays, and so on.

\subsection{Advantages and disadvantages}

There does not appear to be a similar tracking tool available in the literature or practice for comparison, however the advantage of the developed tracking tool overall is two-fold. Firstly, it can quickly adjust the schedule of the remaining months of the planning horizon when there is any change on demand for providers. Secondly, it provides upper and 
lower bounds for the number of workdays which make it very flexible for the users to implement the results. Whilst the tool is dependent on monthly data entry, the availability of current and comprehensive data is integral to accurate scheduling.

\section{Conclusions}

The proposed provider time allotment tracking tool was successfully implemented in the MCDCM. The tool runs at the beginning of each month; based on the changes on the scheduled plan in the previous month, the tool provides the recommended workdays along with upper and lower bounds for each assignment and each provider for the remaining months of the year. This tool prospectively informs the MSAC team so that adjustments can be made in a timely manner to balance provider assignments. The designed tool has an Excel-based user interface which makes the implementation straightforward.

\section{CONFlicts OF INTEREST Disclosure}

The authors declare they have no conflicts of interest.

\section{REFERENCES}

[1] Bastos LS, Marchesi JF, Hamacher S, et al. A mixed integer programming approach to the patient admission scheduling problem. European Journal of Operational Research. 2019; 273(3): 831-840. https://doi.org/10.1016/j.ejor.2018.09.003

[2] Merrild P. The Biggest U.S. Health Care Challenges Are Management Challenges. Harvard Business Review. 2015. Available from: https://hbr.org/2015/02/the-biggest-u-s-healt $\mathrm{h}$-care-challenges-are-management-challenges

[3] West CP, Dyrbye LN, Shanafelt TD. Physician burnout: contributors, consequences and solutions. Journal of Internal Medicine. 2018; 283(6): 516-529. PMid: 29505159. https ://doi.org/10.1111/ joim. 12752

[4] Green LV. Capacity planning and management in hospitals. In Operations research and health care. Springer; 2005. 15-41 p. https : //doi.org/10.1007/1-4020-8066-2_2

[5] Hans EW, Van Houdenhoven M, Hulshof PJ. A framework for healthcare planning and control. In Handbook of healthcare system scheduling. Springer; 2012. 303-320 p. https ://doi.org/10.1007/97 8-1-4614-1734-7_12

[6] Szabo S, Ferencz V, Pucihar A. Trust, Innovation and Prosperity. Quality Innovation Prosperity. 2013; 17(2): 1-8. https://doi.or g/10.12776/qip.v17i2.224

[7] Lau HC. On the complexity of manpower shift scheduling. Computers \& Operations Research. 1996; 23(1): 93-102. https://doi .or g/10.1016/0305-0548(94)00094-0

[8] Marchesi JF, Hamacher S, Fleck JL. A stochastic programming approach to the physician staffing and scheduling problem. Computers \& Industrial Engineering. 2020; 142: 106281. https://doi.org/ 10.1016/j.cie. 2020.106281

[9] El-Rifai O, Garaix T, Augusto V, et al. A stochastic optimization model for shift scheduling in emergency departments. Health Care Manag Sci. 2015; 18(3): 289-302. PMid: 25270574. https : //doi.org/10.1007/s10729-014-9300-4

[10] Fugener A, Schiffels S, Kolisch R. Overutilization and underutilization of operating rooms - insights from behavioral health care operations management. Health Care Manag Sci. 2017; 20(1): 115-
128. PMid: 26433372. https ://doi.org/10.1007/s10729-015 $-9343-1$

[11] Schutz HJ, Kolisch R. Capacity allocation for demand of different customer-product-combinations with cancellations, no-shows, and overbooking when there is a sequential delivery of service. Annals of Operations Research. 2013; 206(1): 401-423. https : //doi.org/10.1007/s10479-013-1324-5

[12] Daldoul D, Nouaouri I, Bouchriha H, et al. A stochastic model to minimize patient waiting time in an emergency department. Operations Research for Health Care. 2018; 18: 16-25. https : //doi.org/10.1016/j.orhc.2018.01.008

[13] Erhard M, Schoenfelder J, Fugener A, et al. State of the art in physician scheduling. European Journal of Operational Research. 2018; 265(1): 1-18. https://doi.org/10.1016/j.ejor.2017.06.0 37

[14] Alfares HK. Survey, categorization, and comparison of recent tour scheduling literature. Annals of Operations Research. 2004; 127(1): 145-175. https://doi.org/10.1023/B:ANOR.0000019088.98 $647 . \mathrm{e} 2$

[15] Baker KR. Workforce allocation in cyclical scheduling problems: A survey. Journal of the Operational Research Society. 1976; 27(1): 155-167. https ://doi.org/10.1057/jors. 1976.30

[16] Bechtold SE, Brusco MJ, Showalter MJ. A comparative evaluation of labor tour scheduling methods. Decision Sciences. 1991; 22(4): 683-699. https://doi.org/10.1111/j.1540-5915.19 91.tb00359. $\mathrm{x}$

[17] Demirovic E, Musliu N, Winter F. Modeling and solving staff scheduling with partial weighted maxSAT. Annals of Operations Research. 2019; 275(1): 79-99. PMid: 30880860. https://doi.org/10.1 007/s10479-017-2693-y

[18] Ernst AT, Jiang H, Krishnamoorthy M, et al. Staff scheduling and rostering: A review of applications, methods and models. European Journal of Operational Research. 2004; 153(1): 3-27. https : //doi.org/10.1016/S0377-2217(03)00095-X

[19] Van den Bergh J, Beliën J, De Bruecker P, et al. Personnel scheduling: A literature review. European Journal of Operational Research. 2013; 226(3): 367-385. https://doi.org/10.1016/j.ejor.2012.11 .029 